\title{
Bulk plasmon polariton-gap soliton-induced transparency in one-dimensional Kerr-metamaterial superlattices
}

\author{
S. B. Cavalcanti, ${ }^{1, *}$ P. A. Brandão, ${ }^{2}$ A. Bruno-Alfonso, ${ }^{3}$ and L. E. Oliveira ${ }^{2}$ \\ ${ }^{1}$ Instituto de Física, Universidade Federal de Alagoas, Maceió-AL, 57072-970, Brazil \\ ${ }^{2}$ Instituto de Física, Universidade Estadual de Campinas-UNICAMP, Campinas-SP, 13083-859, Brazil \\ ${ }^{3}$ Departamento de Matemática, Faculdade de Ciências, UNESP_Universidade Estadual Paulista, 17033-360, Bauru, SP, Brazil \\ *Corresponding author: sbessa@gmail.com
}

Received October 22, 2013; revised November 29, 2013; accepted November 29, 2013;

posted December 3, 2013 (Doc. ID 199736); published December 24, 2013

\begin{abstract}
We have performed a theoretical study of various arrangements of one-dimensional heterostructures composed by bilayers made of nondispersive (A)/dispersive linear (B) materials and illuminated by an obliquely incident electromagnetic wave, which are shown to exhibit a robust bulk-like plasmon-polariton gap for frequencies below the plasma frequency. The origin of this gap stems from the coupling between photonic and plasmonic modes that may be of a magnetic (electric) origin in a transversal electric (traversal magnetic) configuration yielding a plasmon-polariton mode. By substituting the nondispersive linear layer by a nonlinear Kerr layer, we have found that, for frequencies close to the edge of the plasmon-polariton gap, the transmission of a finite superlattice presents a multistable behavior and it switches from very low values to the maximum transparency at particular values of the incident power. At these frequencies, for those singular points where transmission becomes maximum, we find localized plasmon-polariton-gap solitons of various orders depending on the particular value of the incident power. Present results reveal, therefore, new gap plasmon-soliton solutions that are hybrid modes stemming from the resonant coupling between the incoming electromagnetic wave and the plasmonic modes of the dispersive material, leading to the transparency of a stack with nonlinear inclusions. (C) 2013 Optical Society of America

OCIS codes: (160.1245) Artificially engineered materials; (160.4330) Nonlinear optical materials.

http://dx.doi.org/10.1364/OL.39.000178
\end{abstract}

Wave propagation through one-dimensional (1D) periodic superlattices is characterized by the existence of forbidden frequency bands for which the incident light is completely reflected by the structure. In analogy with electronic band gaps, these so-called Bragg gaps have been widely studied and have their origin attributed to destructive interference. With the advent of metamaterials [1], that is, artificial media with a negative index of refraction, many phenomena in periodic systems have been reported [2-6]. In these materials, also known as left-handed materials (LHM), light phase velocity points to the opposite direction of the group velocity, in contrast with right-handed usual materials (RHM). It follows that by studying light propagation in a superlattice whose unit cell is a RHM/LHM bilayer so designed as to have the same optical path in both layers, one finds yet another type of gap known as the zero $n$ gap. The origin of this gap is due to the fact that, in average, the phase acquired in the RHM is exactly delayed in the LHM, so that there is no light propagation [7-12].

The remarkable electromagnetic properties of metamaterials are determined by the resonant characteristics of the subwavelength plasmonic resonators that compose them. Therefore, the advent of metamaterials has given a new thrust to plasmonic studies due to its potential applications in wave guiding and sensing. Recently, a new type of gap has been reported, in various arrangements of RHM/LHM bilayers, that is, periodic, quasi-periodic, fractal, and disordered superlattices illuminated by obliquely incident electromagnetic wave [13-15]. It is shown that the longitudinal electric field component (magnetic) in a TM (TE) configuration, absent at normal incidence, excites longitudinal bulk-like plasmon-polaritons (PPs).
For frequencies below the plasma frequency, the incoming wave and the backward plasmonic wave are exactly out of phase so that they interfere destructively and the propagation of the incoming wave is hindered. This new PP gap may be much wider than the zero- $n$ gap and is due to the appearance of longitudinal bulk-like PP modes of electric (magnetic) nature in a TM (TE) configuration.

The zero- $n$ gap and the PP gap are of a completely different nature. The zero- $n$ gap is located at lower frequencies. In the particular system that we are dealing with here, the zero- $n$ gap opens at $f=3.55 \mathrm{GHz}$, while the PP gap opens around $f=5.08 \mathrm{GHz}$. Moreover, in contrast with the zero- $n$ gap, the PP gap depends critically on the incidence angle, as it stems from the excitation of longitudinal plasmonic modes along propagation. Hence, there must be an electrical or magnetic component of the propagating eletromagnetic field in this direction to observe this gap. It should be noted here that the PP gap has been predicted even for a single bilayer including loss. This is not the case for the zero- $n$ gap, as it can be verified in [16]. Therefore, it should be easier to implement an experiment that provides the evidence for this new gap.

All that has been said above is focused on the linear propagation regime in the sense that the medium responses are independent of the intensity of the incident field. The nonlinear propagation regime should lead to a much more complex response of the medium to the radiation [17] such as multistability properties of the transmission function depending on the incident field intensity. By multistability here, we mean many possible output values for a single input. As expected, studies on 
layered systems, including nonlinear layers, have been carried out with novel results. For example, introducing RHM nonlinear media in 1D superlattices, by alternating linear/nonlinear media, Bragg-gap soliton solutions have been found, for frequencies at the edge of the linear gap. Soliton-mediated transparency switching, from a state of no transparency at the linear regime to total transparency in the nonlinear regime and also bistability, have been reported $[18,19]$. The same system has been investigated in the case of oblique incidence and soliton-like distribution in the layers are obtained due to the excitation of supermodes [20]. Alternate stacks of nonlinear/linear LHM have also reported the existence of a zero- $n$ gap soliton exhibiting different behavior from the Bragg gap soliton: Hedge and Winful [21,22] have shown that, in the presence of a Kerr nonlinearity, the zero- $n$ gap at the frequency may switch from low transmission to a perfectly transmitting state, forming a nonlinear resonance or gap soliton in the process. Moreover, they suggested that this zero- $n$ gap soliton is essentially omnidirectional, in contrast to the Bragg gap soliton of positive-index periodic structures. Bistable robust behavior and soliton shape preservation have been observed for even relatively high losses [21,22].

As it is well known that nonlinear media enhances substantially plasmonic activity, it is a natural question, therefore, to ask about PP-gap soliton solutions and multistability phenomenon at the edge of a PP gap. The nonlinear layers modulate the transmission via an intensity dependent refractive index that introduces a nonlinear phase shift of the wave traveling along the nonlinear layer. Under suitable conditions, plasmonic modes may couple with the incident wave leading to resonant transmission for particular values of the incident wave intensity. It should be noted here that the control of a multistable system should reveal itself quite useful for the development of ultrafast optical switching devices.

Besides multistable transmission behavior, in the following we show the existence of localized waves within the PP band gap, and refer to them as plasmon-polariton gap solitons. To this end, let us begin by considering a finite superlattice of $N=32$ bilayers periodically composed by nonlinear(A)/linear-LHM layers(B) with the same widths, that is, $d_{A}=d_{B}=10 \mathrm{~mm}$, and study its transmission properties. The main objective here is to investigate theoretically, for what we believe is the first time, multistability and the existence of longitudinal bulk-like PP soliton formation near the band edge of a PP gap. The multilayer is stacked along the $z$-axis direction, and it is surrounded by air. We consider the oblique incidence of TE waves polarized along the $y$-axis direction so that the propagation of the $z$-dependent amplitude of the electric field is governed by

$$
-\frac{d}{d z} \frac{1}{\mu} \frac{d E}{d z}=\left[\frac{\omega^{2} \epsilon}{c^{2}}-\frac{q^{2}}{\mu}\right] E .
$$

Here, $\epsilon$ and $\mu$ are the position-dependent values of the dielectric permittivity and the magnetic permeability, respectively. Moreover, $q$ is the wave-vector component along the $x$ axis, which may be written in terms of the incidence angle $\theta$ within each layer as $q=\omega \beta / c$, with $\beta=n \sin \theta$, where $n$ is the refractive index. The fact that $\beta$ is a constant along the multilayer system is broadly known as the Snell's law.

In the present study, we have used the material properties of the nonlinear layer A and metamaterial layer B as chosen by Hedge and Winful $[\underline{21}, \underline{22}]$ :

$$
\begin{gathered}
\epsilon_{\mathrm{A}}=\epsilon_{0}+a|E|^{2}, \\
\mu_{\mathrm{A}}=\mu_{0}, \\
\epsilon_{\mathrm{B}}=1.6+\frac{40}{0.81-f^{2}-\text { if } \gamma}, \\
\mu_{\mathrm{B}}=1.0+\frac{25}{0.814-f^{2}-\text { if } \gamma},
\end{gathered}
$$

where the frequency $f$ is given in $\mathrm{GHz}$ and $\gamma$ accounts for absorptive loss. The calculations presented here have been performed by using a procedure similar to that outlined by Peschel et al. [23] and Trutschel and Lederer [24]. The above equation for the amplitude of the electric field may be solved by matching the general solutions that apply in each layer, i.e., the continuity of both $E$ and $1 / \mu d E / d z$. After introducing a dimensionless position $\zeta=\omega z / c$, the equation within a homogeneous slab is given by

$$
\frac{d^{2} E}{d \zeta^{2}}+\left(\varepsilon \mu-\beta^{2}\right) E=0
$$

One may solve the above equation for the layered structures of interest, i.e., alternate linear and nonlinear media, and straightforwardly obtain the transmission coefficient $T$. One notes that the transfer function will be linear or nonlinear, depending on the nature of the layer. For a nonlinear layer, we use the Runge-Kutta method whereas a linear layer may be treated by the transfer-matrix technique. In this way, it is possible to determine the values of $T$ that correspond to a chosen value of $a\left|E_{i}\right|^{2}$, where $\left|E_{i}\right|$ is the field amplitude of the incident wave.

We begin by focusing the attention within the frequency region where the linear theory predicts a longitudinal bulk-like PP gap $[\underline{13}, \underline{14}]$ and investigate the switching on the transmission properties by increasing the incident power. We choose an incidence angle of $\theta=$ $\pi / 24$ and plot in Fig. 1 the transmission of an electromagnetic wave in a TE configuration for a frequency band near the lower edge of the PP gap, in the case that $a=$ 0 in Eq. (2a). The frequency at which the layer-B magnetic permeability $\mu_{B}$ vanishes is $f=5.08075 \mathrm{GHz}$ [cf. Eq. (2d)], that is, the frequency around which the PP gap opens up $[13,14]$. In order to see whether the power increase should induce transmission switching and multistability, we turn to Fig. 2 , where we have depicted the 


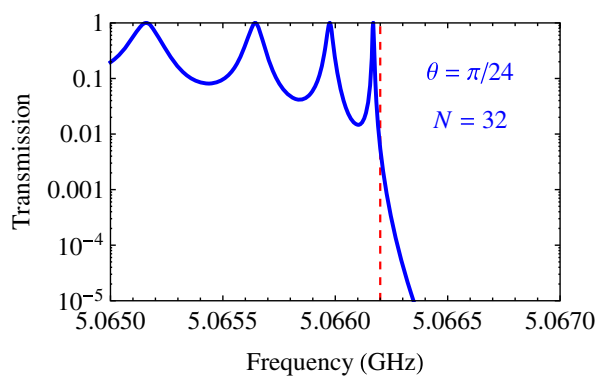

Fig. 1. Transmission through a periodic lossless $(\gamma=0)$ structure composed by $32 \mathrm{AB}$ bilayers (A and $\mathrm{B}$ with equal widths of $10 \mathrm{~mm}$ ), for an incidence angle $\theta=\pi / 24$. The following parameters were chosen: for the linear [ $a=0$ in Eq. (2a)] RHM layer, $\epsilon_{\mathrm{A}}=2.0, \mu_{\mathrm{A}}=1.0$, and, for the linear LHM, the parameters are the same as in the study by Hedge and Winful $[21,22]$ [cf. Eqs. (2c) and (2d)]. The red vertical line is at $f=5.06 \overline{62} \mathrm{GHz}$, for which $T \approx 0.0056$ (see text).

transmission as a function of increasing power fixing $f=5.0662 \mathrm{GHz}$, where $T \approx 0.0056$ for a defocusing nonlinearity, i.e., for $a<0$. For this particular frequency, the transmission switches to one at various discrete values of the field intensity, for low as well as for high intensities, i.e., increasing power leads to several transparency points, occurring at values of $a\left|E_{i}\right|^{2}$ equal to -0.00003 , $-0.0044,-0.022,-0.064,-0.14,-0.24$, and -0.36 [cf. arrows in Fig. 2(a)]. In particular, higher values of intensity lead not only to bistability, but also to a richer structure with increasing multistable output. As we shall demonstrate, this leads to the conclusion that longitudinal bulk-like PP-gap soliton nonlinear discrete resonant modes are excited within the structure.

Let us then choose those particular states of total transmission in Fig. 2 and plot the corresponding spatial profiles of the normalized electric field in Figs. 3 and 4 . The multistability exhibited in the transmission plot reveals itself in the spatial profiles of the field as multisoliton-like distribution. Thus, beginning from the lowest resonant $(T=1)$ intensity, one finds one-soliton, two-soliton, ..., $n$-soliton like profiles by increasing the power in a discrete manner. Figure 3 shows up to the three-soliton distribution, whereas Fig. $\underline{4}$ displays the four-, five-, six-, and seven-soliton distributions. Therefore, one may conclude that the transmission resonance

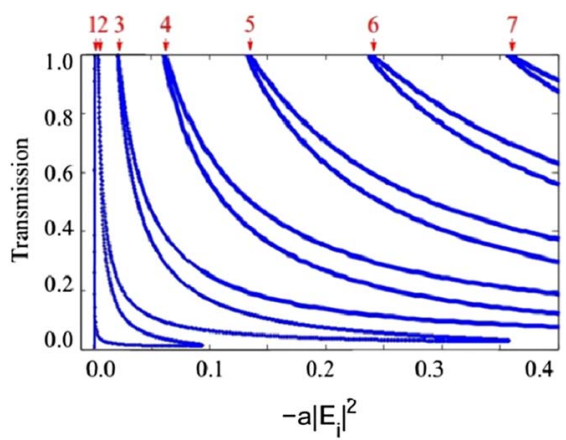

Fig. 2. Transmission through the same structure and linear parameters as in Fig. 1, as a function of increasing power of a defocusing nonlinearity. Several transparency (transmission $T=1$ ) points for $f=5.0662 \mathrm{GHz}$, occurring at values (see arrows) of $a\left|E_{i}\right|^{2}$ equal to $-0.00003,-0.0044,-0.022,-0.064$, $-0.14,-0.24$, and -0.36 .

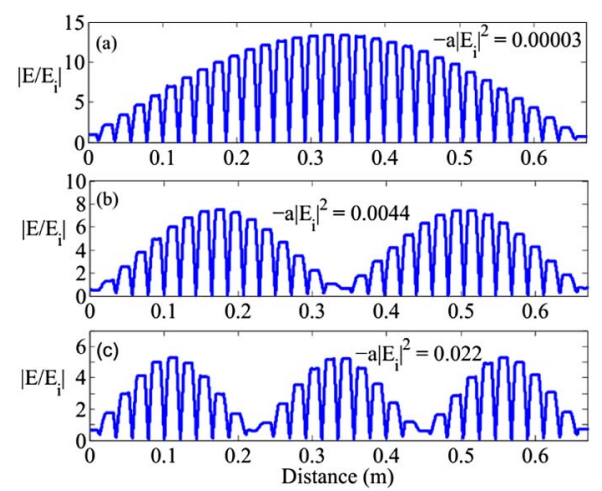

Fig. 3. Spatial profile for the electric field for the lowest three intensities where $T=1$ (cf. Fig. 2). In (a), (b), and (c) there are one, two, and three stable branches, respectively.

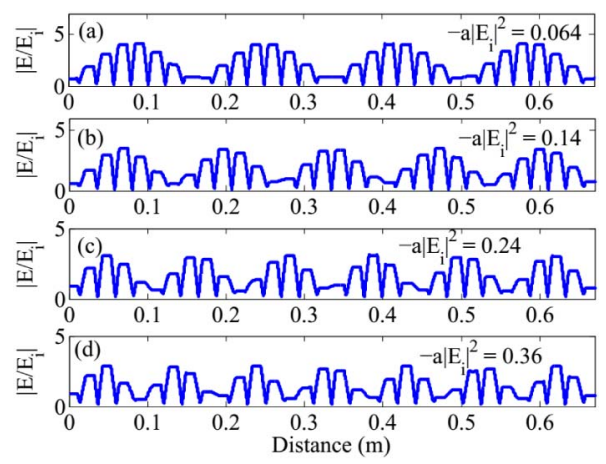

Fig. 4. As in Fig. 3, for the next four higher intensities where $T=1$. In (a), (b), ( $\bar{c})$, and (d), there are four, five, six, and seven stable branches, respectively.

stems from the resonant excitation of PP-gap soliton modes by the incoming wave.

To consider the impact of absorptive loss [25] on the existence of the PP-plasmon-soliton, we turn to Fig. $\underline{5}$, where the electric field profile is plotted as a function of distance for two values of $\gamma$. A fundamental solitary wave is depicted in Fig. 5 for $\gamma=0$ and for $\gamma=10^{-5}$. We see that, although the soliton shrinks dramatically, its shape is preserved just as a typical dissipative solitary wave. Calculations (not shown here) indicate that, for higher field intensities, the higher-order soliton modes survive against relatively high losses. Nowadays, various groups worldwide [2-6] are working toward the

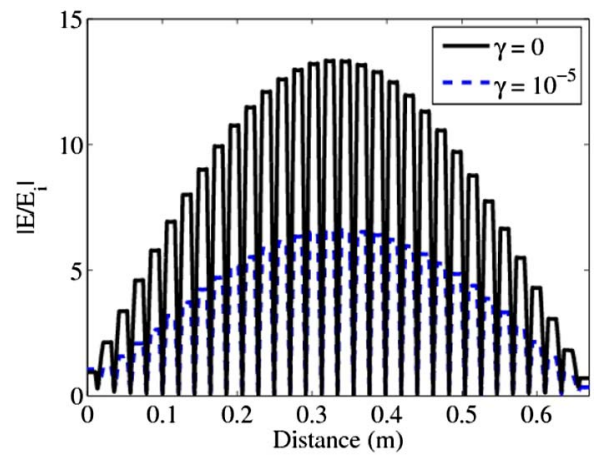

Fig. 5. Plasmon polariton-gap solitons for the case of $\gamma=0$ [no loss, solid curve, cf. Fig. 3(a)] compared with the result for a $\gamma=10^{-5}$ lossy structure (dashed line). 
development of fabrication techniques that compensate losses in metamaterial media and hence produce more efficient plasmonic media: Inclusion of gain material in the areas of intense local fields of a metamaterial demonstrates that it is possible to produce extremely low loss and active optical negative index materials. Moreover, emerging graphene technology should provide low-loss plasmonic materials appropriate to optical devices and the electronic industry. We do believe, therefore, that the present proposed PP-gap soliton induced transparency could be observed in such 1D Kerr-metamaterial superlattices in the near future, as experimental techniques improve so as to cope with the problem of losses.

To conclude, we have presented PP-gap soliton solutions produced by the oblique incidence of an electromagnetic field onto a finite superlattice composed of Kerr-metamaterial bilayers. For frequencies close to the edge of a PP gap, we find PP-induced transparency in the sense that the transparency of the nonlinear stack is induced by the excitation of resonant nonlinear PP-gap soliton modes, i.e., hybrid modes stemming from the coupling between the incoming wave and the plasmonic modes. Finally, we note that the control of the coupling between light and plasmonic modes should find many applications in a wide range of fields, such as sensing, medicine, biophotonics, chemistry, and, most of all, in the area of optical communications, where plasmonic technology holds a promising future.

The present work was partially financed by Brazilian Agencies CNPq, FAPESP (Proc. 2012/51691-0), and FAEPEX-UNICAMP.

\section{References and Notes}

1. V. G. Veselago, Sov. Phys. Usp. 10, 509 (1968).

2. N. I. Zheludev, 2010 Science 328, 582 (2010).

3. A. Fang, T. Koschny, and C. M. Soukoulis, J. Opt. 12, 024013 (2010).
4. S. Xiao, V. P. Drachev, A. V. Kildishev, X. Ni, U. K. Chettiar, H.-K. Yuan, and V. M. Shalaev, Nature 466, 735 (2010).

5. A. Boltasseva and H. A. Atwater, Science 331, 290 (2011).

6. N. I. Zheludev, Opt. Photon. News 22(3), 30 (2011).

7. D. R. Smith, W. J. Padilla, D. C. Vier, S. C. Nemat-Nasser, and S. Schultz, Phys. Rev. Lett. 84, 4184 (2000).

8. J. Li, L. Zhou, C. T. Chan, and P. Sheng, Phys. Rev. Lett. 90, 083901 (2003).

9. H. Jiang, H. Chen, H. Li, Y. Zhang, and S. Zhu, Appl. Phys. Lett. 83, 5386 (2003)

10. M. Liscidini and L. C. Andreani, Phys. Rev. E 73, 016613 (2006).

11. S. Kocaman, R. Chatterjee, N. C. Panoiu, J. F. McMillan, R. M. Osgood, D. L. Kwong, and C. W. Wong, Phys. Rev. Lett. 102, 203905 (2009).

12. J. Schilling, Nat. Photonics 5, 449 (2011).

13. E. Reyes-Gómez, D. Mogilevtsev, S. B. Cavalcanti, C. A. A. Carvalho, and L. E. Oliveira, Europhys. Lett. 88, 24002 (2009).

14. C. A. A. de Carvalho, S. B. Cavalcanti, E. Reyes-Gómez, and L. E. Oliveira, Phys. Rev. B 83, 081408(R) (2011).

15. E. Reyes-Gómez, A. Bruno-Alfonso, S. B. Cavalcanti, and L. E. Oliveira, Phys. Rev. B 85, 195110 (2012).

16. E. Reyes-Gómez, S. B. Cavalcanti, and L. E. Oliveira, Superlattices Microstruct. 64, 590 (2013).

17. Y. V. Kartashov, B. A. Malomed, and L. Torner, Rev. Mod. Phys. 83, 247 (2011).

18. W. Chen and D. L. Mills, Phys. Rev. Lett. 58, 160 (1987).

19. W. Chen and D. L. Mills, Phys. Rev. B 36, 6269 (1987).

20. S. D. Gupta, J. Opt. Soc. Am. B 6, 1927 (1989).

21. R. S. Hedge and H. G. Winful, Microwave and Opt. Tech. Lett. 46, 528 (2005).

22. R. S. Hedge and H. G. Winful, Opt. Lett. 30, 1852 (2005).

23. T. Peschel, P. Dannberg, U. Langbein, and F. Lederer, J. Opt. Soc. Am. B 5, 29 (1988).

24. U. Trutschel and F. Lederer, J. Opt. Soc. Am. B 5, 2530 (1988).

25. A thorough study of the effect of absorptive losses will be published elsewhere. 